Pacific Journal of Mathematics

TENSOR PRODUCTS OF FUNCTION RINGS UNDER 


\title{
TENSOR PRODUCTS OF FUNCTION RINGS UNDER COMPOSITION
}

\author{
N. J. Fine
}

Let $C(X), C(Y)$ be the rings of real-valued continuous functions on the completely regular Hausdorff spaces $X, Y$ and let $T=C(X) \otimes C(Y)$ be the subring of $C(X \times Y)$ generated by functions of the form $f g$, where $f \in C(X)$ and $g \in C(Y)$. If $P$ is a real polynomial, then $P \circ t \in T$ for every $t \in T$. If $G \circ t \in T$ for all $t \in T$ and if $G$ is analytic, then $G$ is a polynomial, provided that $X$ and $Y$ are both infinite (A. W. Hager, Math. Zeitschr. 92, (1966), 210-224, Prop. 3.). In this note I remove the condition of analyticity. Clearly the cardinality condition is necessary, for if either $X$ or $Y$ is finite, then $T=C(X \times Y)$ and $G \circ t \in T$ for every continuous $G$ and for every $t \in T$.

It is convenient to admit a somewhat wider class of $G$ 's. Let $T^{*}=T+i T$, that is, the set of all functions $t_{1}+i t_{2}$ with $t_{1}, t_{2} \in T . \quad\left(T^{*}\right.$ is the tensor product of the complex-valued continuous function rings on $X$ and $Y)$. Define $K(X, Y)$ as the set of all continuous complex-valued functions $G$ on $R$ (the reals) with the property that $G \circ t \in T^{*}$ for all $t \in T$. Then the result is

THEOREM. If $X$ and $Y$ are infinite completely regular Hausdorff spaces, then $K(X, Y)$ consists of all the polynomials with complex coefficients.

It follows from the Theorem that if $G \circ t \in T$ for all $t \in T$, then $G$ is a polynomial with real coefficients.

The proof of the Theorem, which is rather lengthy, will be broken up into a sequence of lemmas.

Lemma 1. Let $\varphi$ and $\psi$ be continuous mappings of $X$ and $Y$ onto $X^{\prime}$ and $Y^{\prime}$ respectively. Then $K(X, Y) \subset K\left(X^{\prime}, Y^{\prime}\right)$.

Proof. Let $G \in K(X, Y), t^{\prime} \in T^{\prime}=C\left(X^{\prime}\right) \otimes C\left(Y^{\prime}\right)$.

I must show that $G \circ t^{\prime} \in T^{* *}$. Define $t$ by

$$
t(x, y)=t^{\prime}(\varphi(x), \psi(y)) \quad(x \in X, y \in Y)
$$

Clearly $t \in T$, and by hypothesis $G \circ t \in T^{*}$. That is, there are continuous complex-valued functions $u_{1}, \cdots, u_{n}$ on $X, v_{1}, \cdots, v_{n}$ on $Y$, such that 


$$
\left(G \circ t^{\prime}\right)(\varphi(x), \psi(y))=\sum_{j=1}^{n} u_{l}(x) v_{l}(y) \quad(x \in X, y \in Y)
$$

If $y_{0}, y_{1}, \cdots, y_{n}$ are any elements of $Y$, then there exist complex $c_{0}, c_{1}, \cdots, c_{n}$ not all 0 such that

$$
\sum_{j=0}^{n} c_{j}\left(G \circ t^{\prime}\right)\left(\varphi(x), \psi\left(y_{j}\right)\right)=0 \quad(x \in X)
$$

since (1) shows that the $y$-sections of $G \circ t$ are contained in an $n$ dimensional subspace of $C(X)+i C(X)$. Let $y_{0}^{\prime}, \cdots, y_{n}^{\prime}$ be any elements of $Y^{\prime}$, and let $x^{\prime}$ be any element of $X^{\prime}$. Then, since $\varphi$ and $\psi$ are onto, there exist $y_{0}, \cdots, y_{n}$ and $x$ such that

$$
\varphi(x)=x^{\prime}, \quad \psi\left(y_{j}\right)=y_{j}^{\prime} \quad(j=0,1, \cdots, n) .
$$

Insert these values in (2) to get

$$
\sum_{j=0}^{n} c_{l}\left(G \circ t^{\prime}\right)\left(x^{\prime}, y_{j}^{\prime}\right)=0
$$

This means that the $y^{\prime}$-sections of $G \circ t^{\prime}$ are contained in an $n$ dimensional subspace of $C\left(X^{\prime}\right)+i C\left(X^{\prime}\right)$. By Hager ${ }^{1}$, this implies that $G \circ t^{\prime} \in T^{* *}$. Hence $G \in K\left(X^{\prime}, Y^{\prime}\right)$.

Lemma 2. If $X^{\prime} \approx X, Y^{\prime} \approx Y$, then $K\left(X^{\prime}, Y^{\prime}\right)=K(X, Y)$.

Proof. Immediate from Lemma 1.

LEMmA 3. If the conclusion of the Theorem holds for all infinite subspaces $X^{\prime}, Y^{\prime}$ of $R$ then the Theorem holds.

Proof. Every infinite completely regular Hausdorff space can be mapped continuously onto an infinite subset of $R$. Apply Lemma 1 and the hypothesis.

Lemma 4. Suppose that $X_{0}$ and $Y_{0}$ are $C$-embedded in $X$ and $Y$ respectively. Then $K(X, Y) \subset K\left(X_{0}, Y_{0}\right)$.

Proof. Let $G \in K(X, Y), t_{0} \in T_{0}=C\left(X_{0}\right) \otimes C\left(Y_{0}\right)$. Then there is a $t \in T$ such that $t \mid\left(X_{0} \times Y_{0}\right)=t_{0}$, obtained by extending each component of $t_{0}$. By assumption, $G \circ t \in T^{*}$. By restriction, $G \circ t_{0} \in T_{0}^{*}$. Hence $G \in K\left(X_{0}, Y_{0}\right)$. 
LEMMA 5. If $X$ is an infinite subset of $R$, then there is a continuous mapping $\varphi$ of $X$ into $R$ such that $\varphi[X]$ contains the terms of a convergent infinite sequence and its limit.

Proof. If $X$ is unbounded, let $p \in X$ and define

$$
\varphi(x)=\frac{x-p}{1+x^{2}} \quad(x \in X) .
$$

Then $\varphi[X]$ has the required property. If $X$ is bounded, then it contains a countably infinite set $\left\{x_{n}\right\}$ such that $x_{n} \rightarrow q$ (perhaps not in $X$ ). Let $p \in X$ and define

$$
\varphi(x)=(x-q)(x-p) \quad(x \in X) .
$$

Clearly $\varphi\left(x_{n}\right) \rightarrow 0=\varphi(p)$. Also the set $\left\{\varphi\left(x_{n}\right)\right\}$ is infinite. Hence $\varphi[X]$ has the required property.

LEMma 6. Let $X_{0}$ be any one infinite set $\left\{x_{n}\right\}_{n=0}^{\infty}$, with $x_{n} \rightarrow x_{0}$. If $K\left(X_{0}, X_{0}\right)$ consists of the complex polynomials, then the Theorem holds.

Proof. Follows from Lemma 3, Lemma 5, Lemma 4, and the fact that $X_{0}$ is compact, hence $C$-embedded in $\varphi[X]$, and Lemma 2 .

LemmA 7. Let $X_{0}=\left\{j / n^{2}: n \geqq 1,0 \leqq j \leqq M_{n}\right\}$, where $M_{n}$ is a sequence of positive integers satisfying $M_{n} \geqq n(n \geqq 1)$. Let $G \in K\left(X_{0}, X_{0}\right)$, with $X_{0} \subset Z(G)$, the zero-set of $G$. Then there exists an $N$ such that

$$
\frac{M_{n}+1}{n^{2}} \in Z(G) \quad(n>N) .
$$

Proof. Define $t \in T_{0}=C\left(X_{0}\right) \otimes C\left(X_{0}\right)$ by

$$
t(x, y)=x+y \quad\left(x \in X_{0}, y \in X_{0}\right) .
$$

Let $N=\operatorname{rank}(G \circ t)$, i.e., the dimension of the vector-space of $y$-sections of $G \circ t$. If $n>N$, there exist $c_{j}(j=1, \cdots, N+1)$ (possibly depending on $n$ ) not all 0 , such that

$$
\sum_{j=1}^{N+1} c_{j} G\left(x+\frac{j}{n^{2}}\right)=0 \quad\left(x \in X_{0}\right) .
$$

(Note that the arguments 


$$
\frac{j}{n^{2}} \leqq \frac{N+1}{n^{2}} \leqq \frac{n}{n^{2}} \leqq \frac{M_{n}}{n^{2}}
$$

are all in $X_{0}$ ). Let $M$ be the largest $j$ such that $c_{1} \neq 0$, so $1 \leqq M \leqq N+1$ and

$$
\sum_{j=1}^{M} c_{j} G\left(x+\frac{j}{n^{2}}\right)=0 \quad\left(x \in X_{0}\right)
$$

Choose $x=\left(M_{n}+1-M\right) / n^{2}$. Since $M \leqq N+1<n+1 \leqq M_{n}+1, x>$ 0 . Since $M \geqq 1, x \leqq M_{n} / n^{2}$. Hence $x \in X_{0}$. Therefore, from (3),

$$
-c_{M} G\left(\frac{M_{n}+1}{n^{2}}\right)=\sum_{j=1}^{M-1} c_{l} G\left(\frac{M_{n}+1-M+j}{n^{2}}\right) .
$$

Since $\quad M_{n}+1-M+j \geqq n+2-M>n+2-(n+1)=1, \quad$ and $M_{n}+1-M+j \leqq M_{n}+1-M+(M-1)=M_{n}$ for all $j$ such that $1 \leqq j \leqq$ $M-1$, the arguments on the right in (4) are all in $X_{0} \subset Z(G)$. Since $c_{M} \neq 0$,

$$
G\left(\frac{M_{n}+1}{n^{2}}\right)=0 \quad(n>N) .
$$

LEMMA 8. Under the hypothesis of Lemma 7, but with $M_{n}=n$ $(n \geqq 1)$, there is an $\alpha>0$ such that $[0, \alpha] \subset Z(G)$.

Proof. Define

$$
\bar{M}_{n}=\sup \left\{M: G\left(\frac{j}{n^{2}}\right)=0 \quad \text { for } \quad j=0,1, \cdots, M\right\} .
$$

Note that $\bar{M}_{n} \geqq n$. Suppose that $\bar{\alpha} \equiv \lim \left(\bar{M}_{n} / n^{2}\right)=0$. Then there is an infinite sequence $n_{1}<n_{2}<\cdots$ such that

$$
\frac{\bar{M}_{n_{i}}}{n_{i}^{2}} \rightarrow 0
$$

Define $L_{n}=\bar{M}_{n}$ if $n=n_{1}$ for some $i, L_{n}=n$ otherwise. Let

$$
X^{\prime}=\left\{\frac{j}{n^{2}}: 0 \leqq j \leqq L_{n}, n \geqq 1\right\}
$$

Then (i) $X^{\prime} \approx X_{0}$, (ii) $X^{\prime} \subset Z(G)$, (iii) $X^{\prime}$ is of the form prescribed in Lemma 7, since $L_{n} \geqq n$. By (i) and Lemma $2, K\left(X_{0}, X_{0}\right)=K\left(X^{\prime}, X^{\prime}\right)$, so 
$G \in K\left(X^{\prime}, X^{\prime}\right)$. Combining this with (ii), (iii), and Lemma 7, one finds that there is an $N$ such that

$$
\frac{L_{n}+1}{n^{2}} \in Z(G) \quad(n>N) .
$$

In particular, for $n=n_{\imath}>N$,

$$
\frac{\bar{M}_{n}+1}{n^{2}} \in Z(G) \text {. }
$$

This contradicts the definition of $\bar{M}_{n}$. Hence $\bar{\alpha}>0(\bar{\alpha}=+\infty$, possibly).

Clearly the set $B=\left\{j / n^{2}: 0 \leqq j \leqq \bar{M}_{n}, n \geqq 1\right\}$ is dense in $[0, \bar{\alpha})$. Since $B \subset Z(G)$, there exists an $\alpha>0$ such that $[0, \alpha] \subset \bar{B} \subset Z(G)$.

LEMMA 9. Under the hypotheses of Lemma 8, G=0.

Proof. Let $\alpha=\sup \{a:[0, a] \subset Z(G)\}$. By Lemma 8, $\alpha>0$. Suppose $\alpha<\infty$. Let $\xi \geqq 0$. For

$$
t(x, y)=\alpha+\xi(x-y) \quad\left(x, y \in X_{0}\right),
$$

let $\operatorname{rank}(G \circ t)=M_{\xi}$. Define $N_{\xi}=1+\max \left(M_{\xi}, \xi M_{\xi} / \alpha\right)$. For $n \geqq N_{\xi}$, there exist $c_{j}\left(j=0,1, \cdots, M_{\xi}\right)$ not all 0 , such that

$$
\sum_{j=0}^{M_{\xi}} c_{j} G\left(\alpha+\xi\left(x-\frac{j}{n^{2}}\right)\right)=0 \quad\left(x \in X_{0}\right) .
$$

(Note that for $0 \leqq j \leqq M_{\xi}$,

$$
0 \leqq \frac{j}{n^{2}} \leqq \frac{M_{\xi}}{n^{2}}<\frac{N_{\xi}}{n^{2}} \leqq \frac{n}{n^{2}},
$$

so $j / n^{2} \in X_{0}$ ). If $q$ is the least $j$ such that $c_{j} \neq 0$, set $x=$ $(q+1) / n^{2}$. Since $0<q+1 \leqq M_{\xi}+1 \leqq N_{\xi} \leqq n, \quad x \in X_{0}$. For $j=q+$ $1, \cdots, M_{\xi}$, one has $\alpha+\xi\left(x-j / n^{2}\right) \leqq \alpha$ and

$$
\begin{aligned}
\alpha+\xi\left(x-\frac{j}{n^{2}}\right) & \geqq \alpha+\xi\left(\frac{q+1}{n^{2}}-\frac{M_{\xi}}{n^{2}}\right) \\
& \geqq \alpha-\frac{\xi M_{\xi}}{n^{2}} \geqq \alpha-\frac{\xi M_{\xi}}{n} \geqq \alpha-\frac{\xi M_{\xi}}{N_{\xi}} \\
& \geqq \alpha-\frac{\alpha\left(N_{\xi}-1\right)}{N_{\xi}}>0 .
\end{aligned}
$$


Hence $\alpha+\xi\left(x-j / n^{2}\right) \in Z(G)$, and from (5),

$$
G\left(\alpha+\frac{\xi}{n^{2}}\right)=-\frac{1}{c_{q}} \sum_{j=q+1}^{M_{\xi}} c_{j} G\left(\alpha+\xi\left(x-\frac{j}{n^{2}}\right)\right)=0 .
$$

Thus it has been proved that for each $\xi \geqq 0$, there is an $N_{\xi}$ such that

$$
G\left(\alpha+\frac{\xi}{n^{2}}\right)=0 \quad\left(n \geqq N_{\xi}\right) .
$$

For each $N=1,2, \cdots$, define

$$
S_{N}=\left\{\xi \geqq 0: n \geqq N \Rightarrow G\left(\alpha+\frac{\xi}{n^{2}}\right)=0\right\} .
$$

Clearly $S_{N}$ is closed and $[0, \infty)=\cup_{N \geqq 1} S_{N}$. By the Baire category theorem, there is an interval $[u, v] \subset S_{N}$ for some $N \geqq 1$, with $0 \leqq u<$ $v$. That is,

$$
G\left(\alpha+\frac{\xi}{n^{2}}\right)=0 \quad(u \leqq \xi \leqq v, n \geqq N) .
$$

Thus the intervals $\left[\alpha+u / n^{2}, \alpha+v / n^{2}\right]$ are contained in $Z(G)$ for all $n \geqq N$. For sufficiently large $n$, these intervals overlap and fill out an interval $(\alpha, \beta]$, with $\beta>\alpha$. Hence $[0, \beta] \subset Z(G)$. This contradicts the definition of $\alpha$, and shows that $\alpha=\infty$. Hence $G(x)=0$ $(x \geqq 0)$. Finally, the function $G_{1}$ defined by $G_{1}(x)=G(1-x)(x \in R)$ belongs to $K\left(X_{0}, X_{0}\right)$ and $G_{1}(x)=0\left(x \in X_{0}\right)$. By what has just been proved, $G_{1}(x)=0 \quad(x \geqq 0)$, so $G(x)=0 \quad(x \leqq 1)$. Therefore $G=$ 0 . (There is an alternate proof that avoids the use of Baire category).

Lemma 10 . Let $X_{0}=\left\{j / n^{2}: 0 \leqq j \leqq n, n \geqq 1\right\}$, and let $G \in K\left(X_{0}, X_{0}\right)$ satisfy, for some positive $h$ and complex $r$,

$$
G(x+h)=r G(x) \quad\left(x \in X_{0}\right) .
$$

Then $G$ is a constant, and $r=1$ unless that constant is 0 .

Proof. The function $G_{1}$ defined by

$$
G_{1}(x)=G(x+h)-r G(x) \quad(x \in R)
$$

belongs to $K\left(X_{0}, X_{0}\right)$, and $X_{0} \subset Z\left(G_{1}\right)$. By Lemma $9, G_{1}=0$, so 


$$
G(x+h)=r G(x) \quad(x \in R) .
$$

Define $F(x)=G(h x)(x \in R)$. Then $F \in K\left(X_{0}, X_{0}\right)$ and

$$
F(x+1)=r F(x) \quad(x \in R) .
$$

Let $N=\operatorname{rank}(F \circ t)$, where $t(x, y)=x y \quad\left(x, y \in X_{0}\right)$. Then the $N+1$ $y$-sections of $F \circ t$ at $y_{t}=2^{-1}(j=0,1, \cdots, N)$ are linearly dependent (note that $\left.2^{-i}=2^{\prime} /\left(2^{j}\right)^{2} \in X_{0}\right)$. Hence there exist $c_{0}, c_{1}, \cdots, c_{N}$ not all 0 such that

$$
\sum_{j=0}^{N} c_{j} F\left(2^{-j} x\right)=0 \quad\left(x \in X_{0}\right)
$$

As above, (8) holds for all $x \in R$, by Lemma 9. Let $M$ be the least nonnegative integer for which an equation of the form (8) holds for all $x \in R$, with the sum running from 0 to $M$ and the $c_{j}$ not all 0 . Then $c_{M} \neq 0$. If $M=0$, then $F=0$ and therefore $G=0$. For $M>0$, let $q$ be the least $j$ such that $c_{l} \neq 0$. Again, if $q=M$, then $G=0$. Hence one may assume that $q<M$. Thus

$$
\sum_{j=q}^{M} c_{j} F\left(2^{-j} x\right)=0 \quad(x \in R)
$$

with $c_{q} \neq 0, \quad c_{M} \neq 0, q<M$, and $M$ minimal. Replace $x$ by $2^{M} x+$ $2^{M}$. Then

$$
\sum_{j=q}^{M} c_{j} F\left(2^{M-j} x+2^{M-j}\right)=0 \quad(x \in R) .
$$

By (7),

$$
\sum_{j=q}^{M} c_{j} r^{2^{M-j}} F\left(2^{M-j} x\right)=0 \quad(x \in R)
$$

Replacing $x$ by $2^{-M} x$, one gets

$$
\sum_{j=q}^{M} c_{j} r^{2^{M-j}} F\left(2^{-j} x\right)=0 \quad(x \in R) .
$$

Combining (9) and (10), one has

$$
\sum_{j=q}^{M-1} c_{j}\left(r-r^{2^{M-J}}\right) F\left(2^{-\jmath} x\right)=0 \quad(x \in R) .
$$


Because of the minimality of $M$, all the coefficients in (11) must be 0 . Since $c_{q} \neq 0$,

$$
r-r^{2^{M-a}}=0 .
$$

Now $r=0$ implies $G(x+h)=0(x \in R)$, that is, $G=0$. Since $q<M$, $2^{M-q} \geqq 2$, so if $r \neq 0, r^{m}=1$ with $m=2^{M-q}-1 \geqq 1$. It follows that

$$
F(x+m)=r^{m} F(x)=F(x) \quad(x \in R) .
$$

Thus $F$ is periodic. Either $F$ (hence $G$ ) is constant or it has a least positive period $p$. From (9),

$$
\sum_{j=q}^{M} c_{j} F\left(2^{M-j} x\right)=0 \quad(x \in R) .
$$

Therefore

$$
F(x)=-\frac{1}{c_{M}} \sum_{j=q}^{M-1} c_{j} F\left(2^{M-j} x\right) \quad(x \in R) .
$$

Hence

$$
\begin{aligned}
F\left(x+\frac{p}{2}\right) & =-\frac{1}{c_{M}} \sum_{j=q}^{M-1} c_{\jmath} F\left(2^{M-\jmath} x+2^{M-\jmath-1} p\right) \\
& =-\frac{1}{c_{M}} \sum_{\jmath=q}^{M-1} c_{\jmath} F\left(2^{M-j} x\right) \\
& =F(x) \quad(x \in R) .
\end{aligned}
$$

This contradicts the fact that $p$ is the minimal period. Hence $F$ is a constant and so is $G$. If $G \neq 0$, then

$$
G(x)=G(x+h)=r G(x)
$$

implies that $r=1$.

Lemma 11 . Let $X_{0}=\left\{j / n^{2}: 0 \leqq j \leqq n, n \geqq 1\right\}, \quad$ and let $G \in K\left(X_{0}, X_{0}\right)$. Then $G$ is a polynomial.

Proof. Let $N=\operatorname{rank}(G \circ t)$, where

$$
t(x, y)=x+y \quad\left(x, y \in X_{0}\right) .
$$


Then, if one reasons as in Lemma 10 , there is an $M \leqq N$ and $c_{0}, \cdots, c_{M}$, with $c_{M}=1$, such that

$$
\sum_{j=0}^{M} c_{j} G\left(x+\frac{j}{N^{2}}\right)=0 \quad\left(x \in X_{0}\right)
$$

Equation (12) holds for all $x \in R$, by Lemma 9. Define $F(x)=$ $G\left(x / N^{2}\right)(x \in R)$. Then

$$
\sum_{j=0}^{M} c_{j} F(x+j)=\sum_{j=0}^{M} c_{j} G\left(\frac{x}{N^{2}}+\frac{j}{N^{2}}\right)=0 \quad(x \in R) .
$$

One may assume that $M$ is minimal for $F$ in equation (13). Write

$$
\varphi(z)=\sum_{j=0}^{M} c_{l} z^{j}
$$

Then, using the standard notation

$$
(E f)(x)=f(x+1)
$$

one has

$$
(\varphi(E) F)(x)=0 \quad(x \in R) .
$$

Let $r$ be any zero of $\varphi(z)$, so that $\varphi(z)=(z-r) \psi(z)$. Define

$$
J(x)=(\psi(E) F)(x) \quad(x \in R) .
$$

By the minimality of $M, J \neq 0$, and

$$
\begin{aligned}
J(x+1)-r J(x) & =(E-r) J(x) \\
& =(E-r) \psi(E) F(x) \\
& =\varphi(E) F(x)=0 \quad(x \in R) .
\end{aligned}
$$

Since $J \in K\left(X_{0}, X_{0}\right)$ and $J \neq 0$, Lemma 10 yields $r=1$. Thus all zeroes of $\varphi(z)$ are 1 , and

$$
\begin{aligned}
& \varphi(z)=(z-1)^{M}, \\
& (E-1)^{M} F(x)=0 \quad(x \in R) .
\end{aligned}
$$

Note that $M=0$ implies $F=G=0$. Let $P(x)$ be the polynomial of degree $\leqq M-1$ which agrees with $F$ at $x=0,1,2, \cdots, M-1$. Then 


$$
\begin{aligned}
P(0) & =F(0) \\
(E-1) P(0) & =(E-1) F(0), \\
& \ldots \\
(E-1)^{M-1} P(0) & =(E-1)^{M-1} F(0) .
\end{aligned}
$$

Also, because $\operatorname{deg} P \leqq M-1$,

$$
(E-1)^{M} P(x)=0=(E-1)^{M} F(x) \quad(x \in R) .
$$

Now

$$
G_{0}(x)=(E-1)^{M-1}(P(x)-F(x)) \in K\left(X_{0}, X_{0}\right)
$$

and

$$
(E-1) G_{0}(x)=0 \quad(x \in R) .
$$

By Lemma $10, G_{0}(x)=$ constant $=G_{0}(0)=0$. Thus

$$
(E-1)^{M-1} P(x)=(E-1)^{M-1} F(x) \quad(x \in R) .
$$

Continuing by induction, one obtains

$$
(E-1)^{M-\jmath} P(x)=(E-1)^{M-i} F(x) \quad(x \in R)
$$

for $j=1,2, \cdots, M$. Thus

$$
F(x)=P(x) \quad(x \in R) .
$$

Therefore $F$, hence $G$, is a polynomial.

Combination of Lemma 11 and Lemma 6 completes the proof of the Theorem.

Received May 18, 1976.

The Pennsylvania State University

UNIVERSITY PARK, PA 16802 



\section{Pacific Journal of Mathematics}

Vol. 68, No. 1

March, 1977

Richard Julian Bagby, On $L^{p}, L^{q}$ multipliers of Fourier transforms . .......

Robert Beauwens and Jean-Jacques Van Binnebeek, Convergence theorems in

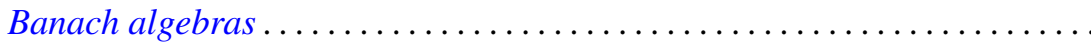

James Cyril Becker, Skew linear vector fields on spheres in the stable

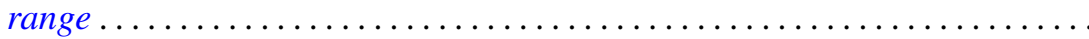

Michael James Beeson, Continuity and comprehension in intuitionistic formal

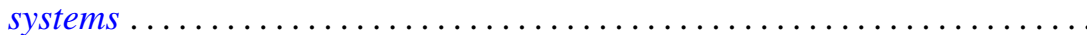

James K. Deveney, Generalized primitive elements for transcendental field

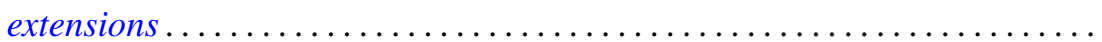

Samuel S. Feder, Samuel Carlos Gitler and K. Y. Lam, Composition properties

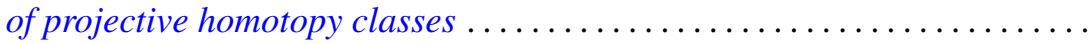

Nathan Jacob Fine, Tensor products of function rings under composition ......

Benno Fuchssteiner, Iterations and fixpoints . . . . . . . . . . . . . .

Wolfgang H. Heil, On punctured balls in manifolds

Shigeru Itoh, A random fixed point theorem for a multivalued contraction

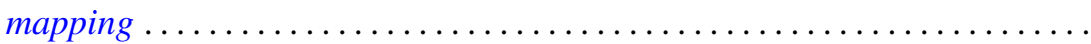

Nicolas P. Jewell, Continuity of module and higher derivations . . . . . . ......

Roger Dale Konyndyk, Residually central wreath products . . . . . . . . . . .

Linda M. Lesniak and John A. Roberts, On Ramsey theory and graphical

parameters.

Vo Thanh Liem, Some cellular subsets of the spheres.

Dieter Lutz, A perturbation theorem for spectral operators

P. H. Maserick, Moments of measures on convex bodies ... . . .

Stephen Joseph McAdam, Unmixed 2-dimensional local domains . .

D. B. McAlister and Norman R. Reilly, E-unitary covers for inverse semigroups...

William H. Meeks, III and Julie Patrusky, Representing codimension-one

homology classes by embedded submanifolds . . .

Premalata Mohapatro, Generalised quasi-Nörlund summability . .

Takahiko Nakazi, Superalgebras of weak-*Dirichlet algebras .

Catherine Louise Olsen, Norms of compact perturbations of operators .

William Henry Ruckle, Absolutely divergent series and isomorphism of

subspaces. II.

Bernard Russo, On the Hausdorff-Young theorem for integral operators .

Arthur Argyle Sagle and J. R. Schumi, Anti-commutative algebras and

homogeneous spaces with multiplications ............

Robert Evert Stong, Stiefel-Whitney classes of manifolds .

D. Suryanarayana, On a theorem of Apostol concerning Möbius functions of

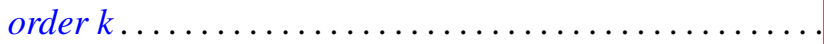

Yoshio Tanaka, On closedness of $C$ - and $C^{*}$-embeddings . . 